\title{
The Impact of Exercise Consultation on Activity Levels and Metabolic Markers in Obese Adolescents: A Pilot Study
}

\author{
M. Henderson, ${ }^{1,2}$ D. Daneman, ${ }^{3}$ C. Huot, ${ }^{2}$ J. McGrath, ${ }^{4}$ M. Lambert, ${ }^{2}$ J. Hux, ${ }^{5}$ \\ G. L. Booth, ${ }^{5,6}$ and A. Hanley,7 \\ ${ }^{1}$ Department of Epidemiology, Biostatistics and Occupational Health, McGill University, Montreal, QC, Canada H3T 1 A2 \\ ${ }^{2}$ Division of Endocrinology, Department of Pediatrics, CHU Ste-Justine and Université de Montréal, 3175 Côte Sainte-Catherine, \\ Montreal, QC, Canada H3T 1C5 \\ ${ }^{3}$ Department of Pediatrics, University of Toronto, Toronto, ON, Canada M5G 1X8 \\ ${ }^{4}$ Department of Psychology, Concordia University, Montreal, QC, Canada H4B 1R6 \\ ${ }^{5}$ Department of Health Policy, Management and Evaluation, University of Toronto, Toronto, ON, Canada M5T 3M6 \\ ${ }^{6}$ Department of Medicine, University of Toronto, Toronto, ON, Canada M5G 2C4 \\ ${ }^{7}$ Department of Nutritional Sciences, University of Toronto, Toronto, ON, Canada M5S 3E2
}

Correspondence should be addressed to M. Henderson, melanie.henderson@mail.mcgill.ca

Received 21 June 2010; Accepted 30 August 2010

Academic Editor: D. A. Schatz

Copyright ( $) 2010$ M. Henderson et al. This is an open access article distributed under the Creative Commons Attribution License, which permits unrestricted use, distribution, and reproduction in any medium, provided the original work is properly cited.

Objective. To assess the impact of exercise consultation on physical activity (PA) levels, anthropometric measures, and metabolic markers in obese adolescents. Methods. Obese adolescents (14-18 years) were randomized to either an exercise consultation (intervention group) or to review "Canada's Physical Activity Guide for Youth" (control group). Outcomes, including accelerometry, anthropometrics, blood pressure, stage of exercise behavior change, fasting glucose, insulin, and lipids, were measured at baseline and 3 months later. Results. Thirty adolescents (mean BMI $=36.1 \mathrm{~kg} / \mathrm{m}^{2} ; \mathrm{SD}=6.9$ ) completed the study. At follow-up, the intervention group had significantly greater PA compared with controls $(P<.05)$. Similarly, the intervention group weighed an average $2.6 \mathrm{~kg}$ less than the control group $(P<.05)$, with a mean BMI z-score of 2.15 compared to 2.21 for controls $(P=.054)$. No other differences were noted. Conclusion. Exercise consultation may be a simple approach to increase PA levels, reduce weight, and lower BMI in obese adolescents.

\section{Introduction}

Obesity rates in Canadian children have tripled over the past three decades [1]. This trend is concerning, given that obese children tend to become obese adults [2-7], putting them at risk for coronary artery disease, type 2 diabetes mellitus, and other chronic conditions [3-8]. Physical activity represents an essential element in energy balance through enhanced energy expenditure. Behavioral lifestyle modification programs have been promising in terms of weight reduction in obese youth $[9,10]$. Previous studies have considered the use of physical activity counseling interventions as an effective and inexpensive method of enhancing physical activity with long-term benefits [11-13].
Exercise consultation is a structured form of physical activity counseling based on the transtheoretical model [14-17], combining both motivational interviewing and cognitive behavioral approaches to promote activity [18]. In a randomized controlled trial involving adults with type 2 diabetes, exercise consultation was associated with a sevenfold increase in activity levels from baseline over a 6-month period [19]. To our knowledge, no study has evaluated this approach in an adolescent population. The purpose of this pilot study was to assess whether exercise consultation would increase physical activity levels, lead to a progression in stage of readiness to change in exercise behavior, as well as improve anthropometric measures and metabolic markers in obese adolescents over a 3-month period. 


\section{Methods and Procedures}

2.1. Participants. Obese adolescents aged 14 to 18 years were recruited over a 9-month period (July 2005 to March 2006) from 4 outpatient clinics at CHU (Centre Hospitalier Universitaire) Ste-Justine (Montreal, Canada), including the endocrinology, diabetes, nutrition, and lipid clinics. For the purpose of this study, obesity was defined as a BMI greater than or equal to the 95th percentile for age (2000 CDC growth charts). Written informed consent was obtained from all participants and their parents. This study received ethics approval from the CHU Ste-Justine Ethics Board. The study was single-blinded, as the subjects were made aware of the two interventions during the process of obtaining consent; individuals involved in data collection and laboratory technicians were blinded to the participants' intervention groups. Based on $80 \%$ power to detect a significant difference in physical activity levels ( $P=.05$, two-sided), we planned on recruiting 23 patients for each study group.

2.2. Study Design. The study timeline is depicted in Figure 1. All participants underwent a baseline assessment that included anthropometric measures (weight, height, waist, and hip circumferences), blood pressure (BP), and selfreported Tanner staging [20]. Patients were then fitted with an Actigraph LS 7164 activity monitor (uniaxial accelerometer, Actigraph LLC, Fort Walton Beach, Florida). They were instructed to wear it under their clothing, fastened to an adjustable elastic belt, during waking hours for a full 7 days. Subjects were advised that the monitors were waterproof, but that they could remove the belt during bathing or swimming activities. Upon their return to the hospital, baseline blood work was drawn after a 12-hour fast and included fasting glucose, insulin, and lipids. Participants also completed the Weekly Activity Checklist questionnaire, which is a 7-day activity recall questionnaire [21], as well as a questionnaire evaluating their stage of change for exercise behavior [22]. The latter categorizes individuals into 5 stages based on their readiness to engage in exercise: stage 1 (precontemplation), stage 2 (contemplation), stage 3 (preparation), stage 4 (action), and stage 5 (maintenance). An index of weekly participation in physical activity was tabulated from the Weekly Activity Checklist by summing participation in all physical activity categories for each participant.

Participants were then randomized to either an exercise consultation (intervention group) or to a review of "Canada's Physical Activity Guide for Youth" (control group). A computer-generated randomization list was drawn up by the statistician. Each participant was allocated the next available number at the time of their second visit (Figure 1).

The standardized exercise consultation consisted of a single one-to-one session designed to educate the participant, strengthen his/her motivation, and develop realistic goals and strategies to promote physical activity customized to the patient's stage of change. For the patient in contemplation, for example, the discussion focused on strategies to move to the preparation stage and incorporate small amounts of physical activity into the participant's daily life. For a patient in the action stage, on the other hand, the discussion focused on strategies to enhance his or her current physical activity levels in a sustainable manner. The consultation was based upon the standardized protocol previously published by Loughlan and Mutrie [18]. We wished to assess the performance of exercise consultation as applied by a physician with no formal training in exercise consultation, other than that acquired by reviewing Loughlan and Mutrie's guidelines, in order to assess the "real life" application of this method.

The control condition included a review and discussion of "Canada's Physical Activity Guide for Youth" [23]. Both conditions were administered by the same researcher and were matched for participant contact time ( 45 minutes duration). Anthropometric measures, blood pressure, Tanner staging, blood work, physical activity, and stage of change were then reassessed 3 months later.

2.3. Biochemical Analyses. All blood samples were processed in the clinical chemistry laboratory of the $\mathrm{CHU}$ Ste-Justine. Plasma glucose was measured enzymatically using glucose oxidase on a Synchron LX20 with Beckman Instruments reagents. Free plasma insulin was measured by radioimmunoassay (KTSP \#11001, Medicorp, Montreal, QC, Canada), after polyethylene glycol precipitation (Diagnostics Products, Los Angeles, CA). Plasma total cholesterol (TC), HDL cholesterol (HDL-C), and triglyceride (TG) concentrations were determined colorimetrically on a Synchron LX20 with Beckman Instruments reagents. TC was measured by the cholesterol oxidase method, HDL-C with a homogeneous assay, and TG by enzymatic hydrolysis followed by the measurement of free glycerol. LDL-C concentrations were calculated according to the Friedewald equation [24]. Insulin sensitivity for each participant was derived from the homeostasis model assessment (HOMA) method using the fasting insulin and fasting glucose values, with the following equation: [fasting insulin $(\mu \mathrm{U} / \mathrm{mL}) \times$ fasting glucose $(\mathrm{mmol} / \mathrm{L})] / 22.5$ [25].

2.4. Accelerometry Data Reduction. In order to ensure that the accelerometer data obtained by participants were valid, we used a number of published methods to ascertain the participants' activity levels using the accelerometry data [2628]. The full activity count was averaged for the actual number of days the unit was worn. As there is no consensus regarding a standardized number of hours to define the daily wearing period $[26,29]$, a minimum of 6 hours was considered a sufficient timeframe for the purpose of this study. While 6 hours remains an arbitrary cutoff, Colley showed [30] that using 6 hours as a minimum wear time ensures that more than $80 \%$ of participants' data are acceptable for analysis. Subjects who did not have adequate data meeting these a priori criteria were excluded from the pertinent analyses. We have included sensitivity analyses using the last value carried forward approach to determine if this impacted our results. A second variable was created using data over a 4-day period. Guidelines for field-based research using accelerometry support the use of 4 days of monitoring, including 3 weekdays and one weekend day, as an adequate reflection of an individual's physical activity 


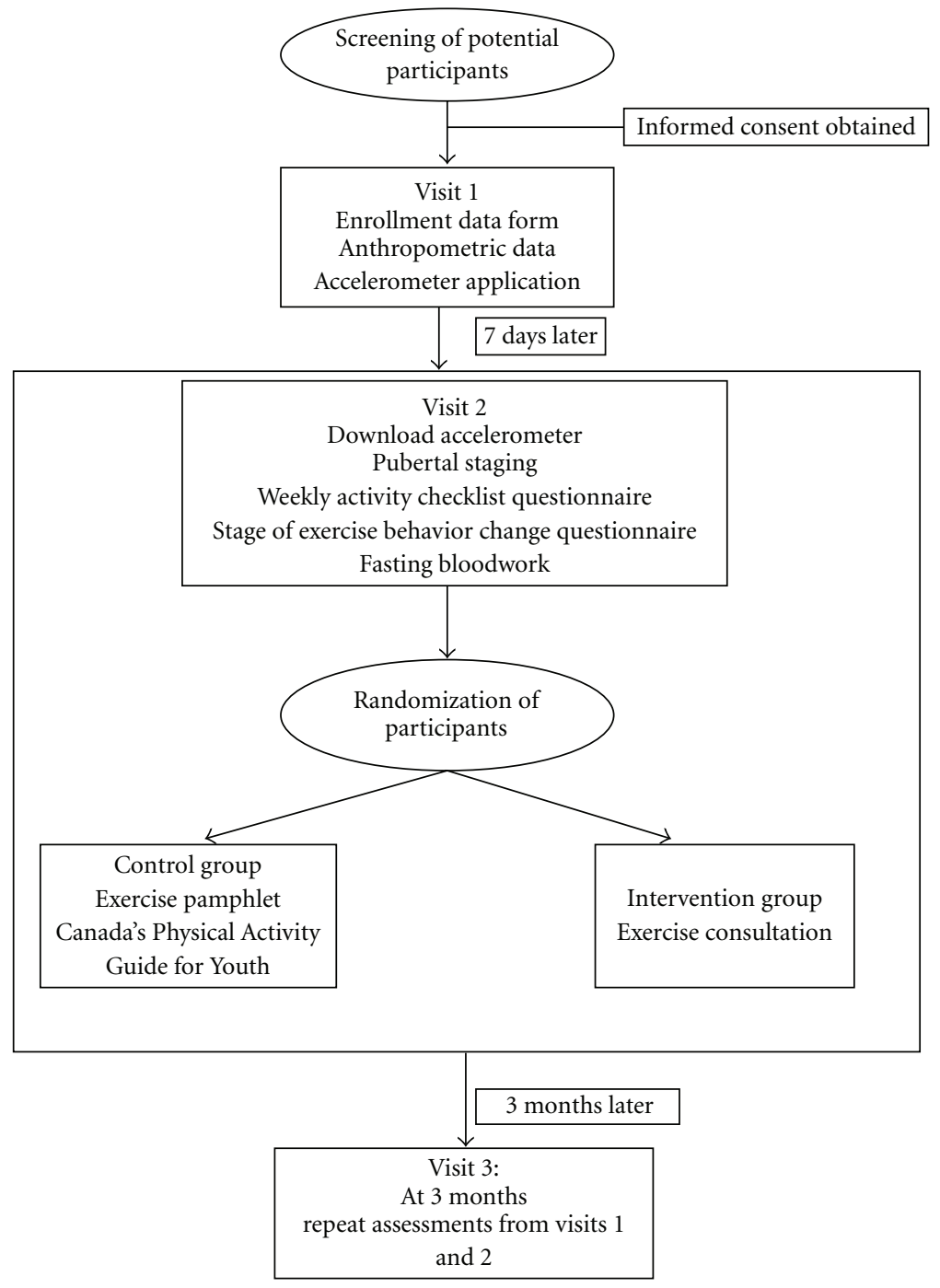

FIgURE 1: Study timeline.

practices [27]. Finally, given that participants did not wear the accelerometer consistently for the entire duration of their waking hours, activity counts per minute were used to try and capture a true reflection of their activity levels [28], in which the total daily counts are divided by the actual number of minutes of wearing, to give a valid estimate of each individual's activity level.

The average daily time spent in sedentary, light, moderate and vigorous physical activity was tabulated for each participant both pre- and postintervention using the data obtained by accelerometry. Cut-points used to define the various levels of intensity were predetermined based on a calibration study by Puyau et al. [31]. Examples of light activities include leisurely walking, warm-up exercises; moderate activities include brisk walking, basketball, and hula hoop; vigorous activities include running, jump rope, and soccer.

2.5. Analyses. An intention-to-treat analytical approach was used for this study. Chi-square and one-way analyses of variance (ANOVA) were used to first evaluate baseline differences between the control and intervention groups.
The effect of the intervention was tested using analyses of covariance (ANCOVA) to control for the baseline values of continuous data. Chi-square tests (or Fisher's exact when appropriate) were used for categorical data. Cohen's $d$ for dependent means was calculated to estimate effect size, with pre- to post differences reported for the intervention group only for simplicity. BMI $\mathrm{z}$-scores were calculated based on CDC 2000 data. All analyses were performed using SPSS 12.0 (SPSS Inc., Chicago, IL, USA).

\section{Results}

Thirty-three adolescents consented to participation (consent rate $43 \%$ of 77 eligible adolescents approached to participate). There were no differences between participants and those who refused to participate in terms of age, sex, or BMI z-score (data not shown). Three participants did not complete the study, yielding an attrition rate of $9.1 \%$. Two of these subjects were prematurely randomized prior to obtaining baseline data. These subjects were discovered to 
be ineligible $(n=1)$ or were not interested in participating $(n=1)$, prior to completing the baseline information step. They were therefore excluded from the analysis given that there was no informative data on them and no intervention had been allocated to them. A third participant dropped out prior to the follow-up visit.

Participant baseline characteristics are presented in Table 1 . No statistically significant between-group differences were noted at baseline for age, sex, anthropometric measures, Tanner stage, stage of change, or accelerometer data. There were no differences across sexes for age, BMI, and BMI zscore. Not unexpectedly, girls had higher hip circumference values and consequently lower waist-to-hip ratios (see Table 1).

3.1. Physical Activity Levels. At the 3-month follow-up, the intervention group had significantly higher physical activity levels than the control group for all accelerometry variables (Table 2$)$ : activity counts per minute $(P<.001, d=0.74)$; all days when the accelerometer was actually worn $(P<.01, d=$ $0.50)$; average activity count over 3 weekdays plus 1 weekend day $(P<.05, d=0.46)$.

The intervention group spent significantly more time in light physical activity compared to the control group at the 3 month follow-up $(P<.001, d=0.70)$. The average increase in time spent in light physical activity was 18 minutes per day for the intervention group while the control group decreased by an average of 6 minutes per day. No significant between-group differences were found for moderate or intense physical activity. The mean duration of wear of the accelerometer, in terms of days worn and hours/day, was not different between the groups both pre- and postintervention. There were no significant differences between groups for the weekly frequency of reported activities from the Activity Recall questionnaire. We performed sensitivity analyses using baseline data carried forward to follow-up data for the participant who dropped out. There were no significant changes to the results presented above. Similarly, in subjects who did not have complete accelerometry data using the a priori criteria previously described, we carried out sensitivity analyses using baseline data carried forward as a conservative approach and found no significant changes to the above reported results.

3.2. Secondary Outcome Measures. At baseline, most participants (44\% of controls and $40 \%$ of the intervention group) were in the "preparation" stage of change of the transtheoretical model (stage 3 out of 5). At the 3 -month follow-up, there was a significant difference in stage of change between the groups. The majority of participants in the control group $(68.8 \%)$ remained in stage 3 while the majority of participants in the intervention group (50\%) moved to stage 4 (Fisher's exact $P<.05, d=1.08$ ).

At the 3-month follow-up, the intervention group had significantly lower weight and BMI z-scores and smaller hip circumferences compared to the control group (Table 2). After controlling for baseline weight, the participants in the intervention group weighed $2.6 \mathrm{~kg}$ less than the control group participants at follow-up $(P<.05, d=0.44)$. The mean BMI z-score at follow-up for the intervention group was $2.15 \mathrm{~kg} / \mathrm{m}^{2}$, compared to $2.21 \mathrm{~kg} / \mathrm{m}^{2}$ for the control group $(P=.054, d=1.03)$. Hip circumference was $3.3 \mathrm{~cm}$ smaller in the intervention group than the control group $(P<.01 ; d=0.57)$. These changes remained significant in the sensitivity analyses performed to take into account the subject lost to follow-up. There were no significant differences noted between the groups for waist circumference, waist-to-hip ratio, systolic blood pressure, diastolic blood pressure, or the metabolic markers (fasting blood glucose, insulin, and lipids).

\section{Discussion}

The need for the development of efficacious and practical counseling approaches to increase physical activity in youth has been previously emphasized [32]. The results of this pilot study suggest that exercise consultation is a promising strategy to increase physical activity levels and decrease weight and BMI in obese adolescents.

Although increases in moderate and vigorous physical activity levels in youth are ultimately the goal to target weight loss, the increase in time spent in light activities of 18 minutes daily for the intervention group may be the first step in reaching these goals. The majority of participants were either in contemplation (stage 2) or preparation (stage 3 ) stages on the stage of change spectrum for physical activity: in other words, participants were either thinking about engaging in more physical activity or engaging in less than 20 minutes of physical activity three times weekly. In this context, an increase of 18 minutes per day of even light physical activity is a promising start. Light-to-moderate and moderate levels of physical activity, such as brisk walking, are known to be associated with health benefits in adults, including decreased risk of type 2 diabetes mellitus and cardiovascular disease [33-37]. Data from the Cardiovascular Risk in Young Finns Study $[38,39]$ and other studies $[40,41]$ suggest that similar benefits in terms of cardiovascular disease prevention derived from moderate intensity physical activity may be seen in children and adolescents. The progression in stage of exercise behavior noted in the intervention group lends further support to the fact that the increase in physical activity captured in this study reflects the initiation of changes in attitudes and lifestyle habits, with an increased readiness to engage in physical activity. Future research should evaluate whether this trend is sustainable over time.

4.1. A Simple Intervention for Primary Care Use. Approximately $80 \%$ of American children and adolescents consult their primary care physician at least once yearly [32]. Consequently, primary care providers could play a major role in the prevention and treatment of childhood obesity. It is estimated that $30 \%-40 \%$ of primary care givers provide some form of counseling on diet and physical activity to young people, although the quality of this counseling remains uncertain [42]. 
TABLE 1: Baseline characteristics of intervention and control group participants.

\begin{tabular}{|c|c|c|}
\hline Participants characteristics & Control $(n=16)$ & Intervention $(n=15)$ \\
\hline Age (years), mean $\pm S D^{a}$ & $15.3 \pm 1.1$ & $16.0 \pm 1.2$ \\
\hline Female, $n(\%)^{\mathrm{a}}$ & $11(68.8 \%)$ & $8(57.1 \%)$ \\
\hline $\mathrm{BMI}\left(\mathrm{Kg} / \mathrm{m}^{2}\right)$, mean $\pm \mathrm{SD}^{\mathrm{a}}$ & $36.4 \pm 7.4$ & $35.7 \pm 6.5$ \\
\hline \multicolumn{3}{|l|}{ Baseline SOC, $n(\%)^{\mathrm{a}}$} \\
\hline Contemplation & $5(31.3 \%)$ & $5(33.3 \%)$ \\
\hline Preparation & $7(43.8 \%)$ & $6(40.0 \%)$ \\
\hline Action & $1(6.3 \%)$ & $1(6.7 \%)$ \\
\hline Maintenance & $3(18.8 \%)$ & $3(20.0 \%)$ \\
\hline \multicolumn{3}{|l|}{ Tanner stage } \\
\hline \multicolumn{3}{|l|}{ Genitalia, $n(\%)^{\mathrm{a}}$} \\
\hline Tanner 1 & 0 & 0 \\
\hline Tanner 2 & 0 & 0 \\
\hline Tanner 3 & $4(25.0 \%)$ & $5(35.7 \%)$ \\
\hline Tanner 4 & $9(56.3 \%)$ & $3(41.4 \%)$ \\
\hline Tanner 5 & $3(18.8 \%)$ & $6(42.9 \%)$ \\
\hline \multicolumn{3}{|l|}{ Tanner stage } \\
\hline \multicolumn{3}{|l|}{ Pubic hair, $n(\%)^{\mathrm{a}}$} \\
\hline Tanner 1 & 0 & $1(6.7 \%)$ \\
\hline Tanner 2 & 0 & 0 \\
\hline Tanner 3 & $3(18.8 \%)$ & $4(26.7 \%)$ \\
\hline Tanner 4 & $4(25.0 \%)$ & $2(13.3 \%)$ \\
\hline Tanner 5 & $9(56.3 \%)$ & $8(53.3 \%)$ \\
\hline Activity counts per minute, mean $\pm \mathrm{SD}^{\mathrm{a}}$ & $293.4(94.4)$ & $339.8(106.8)$ \\
\hline Average Counts all days actually worn, mean $\pm \mathrm{SD}^{\mathrm{a}}$ & $244950.0(69978.5)$ & $275564.3(81064.9)$ \\
\hline Average Activity Counts over 3 week days +1 week-end day, mean $\pm \mathrm{SD}^{\mathrm{a}}$ & $260352.0(74771.6)$ & $291063.1(99417.1)$ \\
\hline Frequency of Activity (questionnaire), mean $\pm \mathrm{SD}^{\mathrm{a}}$ & $9.4(7.2)$ & $8.1(3.6)$ \\
\hline Light activity (minutes/week), mean $\pm \mathrm{SD}^{\mathrm{a}}$ & $77.3(24.4)$ & $84.8(24.5)$ \\
\hline Moderate activity (minutes/week), mean $\pm \mathrm{SD}^{\mathrm{a}}$ & $10.6(10.2)$ & $16.6(10.1)$ \\
\hline Vigorous activity (minutes/week), mean $\pm \mathrm{SD}^{\mathrm{a}}$ & $0.90(3.1)$ & $0.13(0.4)$ \\
\hline Weight $(\mathrm{kg})$, mean $\pm \mathrm{SD}^{\mathrm{a}}$ & $98.0(18.8)$ & $97.5(20.1)$ \\
\hline BMI Z-scores $\left(\mathrm{kg} / \mathrm{m}^{2}\right)$, mean $\pm \mathrm{SD}^{\mathrm{a}}$ & $2.2(0.4)$ & $2.2(0.4)$ \\
\hline Waist circumference $(\mathrm{cm})$, mean $\pm \mathrm{SD}^{\mathrm{a}}$ & $109.0(16.1)$ & $109.7(15.2)$ \\
\hline Hip circumference $(\mathrm{cm})$, mean $\pm \mathrm{SD}^{\mathrm{a}}$ & $114.4(13.7)$ & $115.1(14.0)$ \\
\hline Waist-to-hip ratio, mean $\pm \mathrm{SD}^{\mathrm{a}}$ & $0.95(0.1)$ & $0.96(0.1)$ \\
\hline Wear time baseline (days), mean $\pm \mathrm{SD}^{\mathrm{a}}$ & $6.9(0.28)$ & $6.2(0.97)$ \\
\hline Wear time baseline (hours/day), mean $\pm \mathrm{SD}^{\mathrm{a}}$ & $14.2(0.8)$ & $13.3(1.5)$ \\
\hline
\end{tabular}

$n$ : number of participants; SD: standard deviation; BMI: body mass index; SOC: stage of change.

${ }^{\mathrm{a}}$ No significant differences were observed between the groups.

Lack of patient motivation has been identified as one of the most common barriers to the treatment of obesity in youth [43]. Moreover, physicians are sceptical of their ability to help patients change their behaviours $[43,44]$. Exercise consultation provides a simple counseling approach, tailored to the patient's current attitudes, beliefs, and motivation, to positively promote physical activity. It does not require extensive training, making it a user-friendly tool for clinicians. It can be learned by any member of the treatment team and can be transferred across various clinical settings. Its successful application in this pilot study suggests that it is a feasible intervention that has the potential, at least in the short term, to improve physical activity levels and decrease weight and BMI in obese adolescents, while overcoming some of the obstacles cited by practitioners working with obese children and adolescents. Further evaluation will be required to determine the long-term efficacy and costeffectiveness of exercise consultation. In addition, the impact of repeated consultations over time may provide more sustainable behaviour change, and this could be addressed in future research.

The strengths of this study were its design (a prospective, randomized, single-blind trial), the simple and userfriendly nature of the intervention, and its theoretical framework. Indeed, the presence of a theoretical framework underpinning an exercise intervention has been cited as 
TABLE 2: Comparison of intervention versus control group: accelerometry, anthropometry, and metabolic markers.

\begin{tabular}{|c|c|c|c|c|c|c|c|c|}
\hline \multirow{2}{*}{ Variable } & \multicolumn{3}{|c|}{ Control group } & \multicolumn{3}{|c|}{ Intervention group } & \multirow{2}{*}{$P$} & \multirow{2}{*}{$d$} \\
\hline & Mean & SE & $95 \% \mathrm{CI}$ & Mean & SE & $95 \% \mathrm{CI}$ & & \\
\hline Activity counts per minute & 286.1 & 30.3 & $223.6-348.7$ & 439.4 & 29.2 & $379.2-499.6$ & .001 & 0.74 \\
\hline $\begin{array}{l}\text { Average Counts all days } \\
\text { actually worn }\end{array}$ & 222247.6 & 26519.5 & $167629.8-276865.4$ & 345901.0 & 26519.5 & $291283.2-400518.8$ & .003 & 0.50 \\
\hline $\begin{array}{l}\text { Average Activity Counts } \\
\text { over } 3 \text { week days }+1 \\
\text { week-end day }\end{array}$ & 249640.2 & 22773.8 & 201974.1-297306.3 & 319169.4 & 22773.8 & $271503.3-366835.6$ & .046 & 0.46 \\
\hline $\begin{array}{l}\text { Frequency of Activity } \\
\text { (questionnaire) }\end{array}$ & 14.5 & 1.7 & $10.9-18.0$ & 12.6 & 1.8 & $8.8-16.4$ & .458 & 0.56 \\
\hline $\begin{array}{l}\text { Light activity } \\
\text { (minutes/week) }\end{array}$ & 69.9 & 4.8 & $60.1-79.8$ & 101.1 & 4.6 & $91.7-110.6$ & .0009 & 0.70 \\
\hline $\begin{array}{l}\text { Moderate activity } \\
\text { (minutes/week) }\end{array}$ & 15.2 & 6.2 & $2.5-27.9$ & 24.0 & 5.9 & $11.7-36.2$ & .322 & 0.28 \\
\hline $\begin{array}{l}\text { Vigorous activity } \\
\text { (minutes/week) }\end{array}$ & 0.17 & 0.07 & $0.032-0.314$ & 0.25 & 0.07 & $0.117-0.388$ & .416 & 0.02 \\
\hline Weight (kg) & 98.5 & 0.8 & $96.8-100.1$ & 95.9 & 0.9 & $94.1-97.6$ & .034 & 0.44 \\
\hline BMI z-scores $\left(\mathrm{kg} / \mathrm{m}^{2}\right)$ & 2.21 & 0.022 & $2.17-2.25$ & 2.15 & 0.023 & $2.10-2.19$ & .054 & 1.03 \\
\hline Waist circumference $(\mathrm{cm})$ & 107.4 & 1.22 & $104.9-109.9$ & 107.2 & 1.30 & $104.5-109.9$ & .915 & 0.26 \\
\hline Hip circumference $(\mathrm{cm})$ & 115.6 & 0.69 & $114.2-117.0$ & 112.3 & 0.74 & $110.8-113.8$ & .003 & 0.57 \\
\hline Waist-to-hip ratio & 0.93 & 0.012 & $0.91-0.96$ & 0.96 & 0.013 & $0.93-0.98$ & .169 & 0.12 \\
\hline
\end{tabular}

Marginal means controlling for baseline values are reported above. $d$ : Cohen's $d$ for dependent means (pre- to postdifferences for intervention group only).

essential to developing successful interventions [45]. In addition, the measurement of physical activity levels was done using both a self-report questionnaire and an objective measure, the accelerometer, arguably the best available tool to measure physical activity [26]. A further strength of this study is the target population of obese adolescents. Indeed, few previous exercise interventions in youth have targeted teenagers $[10,45,46]$. This is unfortunate, as it represents an opportune time for intervention, given that physical activity levels have been shown to decline in adolescence, particularly in girls [47].

The significant limitations of the present study include its small sample size and low participation, with a $43 \%$ consent rate (77 potential participants approached, with 33 consenting to participate) and 9\% attrition rate. These figures are consistent with previous work in this patient population, with reported consent rates tending to be modest, ranging from $19 \%-30 \%[48,49]$. The fact that we were able to detect a difference in the intervention group, despite falling short of our initial recruitment objective, strengthens the finding that exercise consultation is promising as a means of increasing physical activity in youth. In addition, the participants in this study were recruited from clinics in a tertiary care center, thus limiting the generalizability of the results to a community care setting. No specific instructions were given to participants in this pilot study regarding dietary habits, and no diet related data were gathered. The possibility that dietary differences could be responsible for differences in weight and BMI cannot be excluded, although dietary confounders should have been distributed evenly between the two groups, given randomization, and therefore are unlikely to explain the results of this study. Finally, the short duration of follow-up remains a limitation in this study: the behaviour change literature suggests that changes are generally unlikely to be sustained in the long term. Therefore, measurements of behaviour modification soon after an intervention may not reflect long-term adherence to lifestyle changes [17]. Further studies are required to ascertain the cost-effectiveness and long-term impact of exercise consultation on activity levels in adolescence. The participants' parents were not included in the consultations with the adolescents. Exploring the role of parental involvement in affecting physical activity levels would also be of interest in a future study.

\section{Conclusion}

Childhood obesity is an epidemic that must be addressed by many disciplines, and health care providers remain the frontline agents of change. Simple, cost-effective strategies are required to foster changes in lifestyle habits, including physical activity. This pilot study confirmed the feasibility of exercise consultation as an easily applicable, user-friendly strategy to promote physical activity and weight loss in obese adolescents. Further studies assessing the efficacy of exercise consultation in other settings, with a larger study sample, with separate researchers consulting the groups, and over a longer time span, will be required in order to determine whether exercise consultation is a strategy that should be included in current guidelines for the treatment of childhood and adolescent obesity.

\section{Competing Interests}

The authors have no conflicts of interest relevants to this paper to disclose. 


\section{Acknowledgments}

This paper was funded by a grant from the Centre de Recherche of the CHU Ste-Justine. The funding sponsor had no role in the study design; the collection, analysis, and interpretation of data; the writing of the report, nor the decision to submit the paper for publication. The authors would like to thank Nathalie Bureau and Renée Dicaire for their devotion to this project. They also wish to thank the adolescents who participated in the study, and their families, for their indispensable contribution.

\section{References}

[1] Statistics Canada, The Daily, catalogue 11-001-XIE, p. 2, July 2005.

[2] R. E. Andersen, "The spread of the childhood obesity epidemic," Canadian Medical Association Journal, vol. 163, no. 11, pp. 1461-1462, 2000.

[3] S. R. Srinivasan, W. Bao, W. A. Wattigney, and G. S. Berenson, "Adolescent overweight is associated with adult overweight and related multiple cardiovascular risk factors: the Bogalusa Heart Study," Metabolism, vol. 45, no. 2, pp. 235-240, 1996.

[4] D. S. Freedman, C. L. Shear, G. L. Burke et al., "Persistence of juvenile-onset obesity over eight years: the Bogalusa Heart Study," American Journal of Public Health, vol. 77, no. 5, pp. 588-592, 1987.

[5] F. E. M. Braddon, B. Rodgers, M. E. J. Wadsworth, and J. M. C. Davies, "Onset of obesity in a 36 year birth cohort study," British Medical Journal, vol. 293, no. 6542, pp. 299-303, 1986.

[6] S. S. Guo, A. F. Roche, W. C. Chumlea, J. D. Gardner, and R. M. Siervogel, "The predictive value of childhood body mass index values for overweight at age $35 \mathrm{y}$," American Journal of Clinical Nutrition, vol. 59, no. 4, pp. 810-819, 1994.

[7] R. C. Whitaker, J. A. Wright, M. S. Pepe, K. D. Seidel, and W. H. Dietz, "Predicting obesity in young adulthood from childhood and parental obesity," The New England Journal of Medicine, vol. 337, no. 13, pp. 869-873, 1997.

[8] National Institutes of Health, "Clinical guidelines on the identification, evaluation, and treatment of overweight and obesity in adults," National Institutes of Health, Bethesda, Md, USA, 1998, http://www.nhlbi.nih.gov/guidelines/ obesity/ob_home.htm.

[9] H. Oude Luttikhuis, L. Baur, H. Jansen et al., "Interventions for treating obesity in children," Cochrane Database of Systematic Reviews, no. 1, Article ID CD001872, 2009.

[10] D. E. Wilfley, T. L. Tibbs, D. J. Van Buren, K. P. Reach, M. S. Walker, and L. H. Epstein, "Lifestyle interventions in the treatment of childhood overweight: a meata-analytic review of randomized controlled trials," Health Psychology, vol. 26, no. 5, pp. 521-532, 2007.

[11] M. Lowther, N. Mutrie, and E. M. Scott, "Promoting physical activity in a socially and economically deprived community: a 12 month randomized control trial of fitness assessment and exercise consultation," Journal of Sports Sciences, vol. 20, no. 7, pp. 577-588, 2002.

[12] S. S. Harris, C. J. Caspersen, G. H. DeFriese, and E. H. Estes Jr., "Physical activity counseling for healthy adults as a primary preventive intervention in the clinical setting. Report for the US Preventive Services Task Force," Journal of the American Medical Association, vol. 261, no. 24, pp. 3588-3598, 1989.

[13] K. J. Calfas, J. F. Sallis, B. Oldenburg, and M. French, "Mediators of change in physical activity following an intervention in primary care: PACE," Preventive Medicine, vol. 26, no. 3, pp. 297-304, 1997.

[14] J. O. Prochaska and C. C. DiClemente, "Stages and processes of self-change of smoking: toward an integrative model of change," Journal of Consulting and Clinical Psychology, vol. 51, no. 3, pp. 390-395, 1983.

[15] J. O. Prochaska, W. F. Velicer, J. S. Rossi et al., "Stages of change and decisional balance for 12 problem behaviors," Health Psychology, vol. 13, no. 1, pp. 39-46, 1994.

[16] B. H. Marcus and L. R. Simkin, "The transtheoretical model: applications to exercise behavior," Medicine and Science in Sports and Exercise, vol. 26, no. 11, pp. 1400-1404, 1994.

[17] J. O. Prochaska and W. F. Velicer, "The transtheoretical model of health behavior change," American Journal of Health Promotion, vol. 12, no. 1, pp. 38-48, 1997.

[18] C. Loughlan and N. Mutrie, "Conducting an exercise consultation: guidelines for health professionals," Journal of the Institute of Health Education, vol. 33, pp. 78-82, 1995.

[19] A. Kirk, P. MacIntyre, N. Mutrie, and M. Fisher, "Increasing physical activity in people with type 2 diabetes," Diabetes Care, vol. 26, no. 4, pp. 1186-1192, 2003.

[20] N. M. Morris and J. R. Udry, "Validation of a self-administered instrument to assess stage of adolescent development," Journal of Youth and Adolescence, vol. 9, no. 3, pp. 271-280, 1980.

[21] J. F. Sallis, S. A. Condon, K. J. Goggin, J. J. Roby, B. Kolody, and J. E. Alcaraz, "The development of self-administered physical activity surveys for 4th grade students," Research Quarterly for Exercise and Sport, vol. 64, no. 1, pp. 25-31, 1993.

[22] B. H. Marcus, V. C. Selby, R. S. Niaura, and J. S. Rossi, "Selfefficacy and the stages of exercise behavior change," Research Quarterly for Exercise and Sport, vol. 63, no. 1, pp. 60-66, 1992.

[23] “Canada's Physical Activity Guide for Youth," August 2010, http://www.phac-aspc.gc.ca/hp-ps/hl-mvs/pag-gap/cy-ej/ index-eng.php.

[24] W. T. Friedewald, R. I. Levy, and D. S. Fredrickson, "Estimation of the concentration of low-density lipoprotein cholesterol in plasma, without use of the preparative ultracentrifuge," Clinical Chemistry, vol. 18, no. 6, pp. 499-502, 1972.

[25] D. R. Matthews, J. P. Hosker, A. S. Rudenski, B. A. Naylor, D. F. Treacher, and R. C. Turner, "Homeostasis model assessment: Insulin resistance and $\beta$-cell function from fasting plasma glucose and insulin concentrations in man," Diabetologia, vol. 28, no. 7, pp. 412-419, 1985.

[26] D. S. Ward, K. R. Evenson, A. Vaughn, A. B. Rodgers, and R. P. Troiano, "Accelerometer use in physical activity: best practices and research recommendations," Medicine and Science in Sports and Exercise, vol. 37, no. 11, supplement, pp. S582S588, 2005.

[27] S. G. Trost, K. L. Mciver, and R. R. Pate, "Conducting accelerometer-based activity assessments in field-based research," Medicine and Science in Sports and Exercise, vol. 37, no. 11, pp. S531-S543, 2005.

[28] P. Freedson, D. Pober, and K. F. Janz, "Calibration of accelerometer output for children," Medicine and Science in Sports and Exercise, vol. 37, no. 11, supplement, pp. S523S530, 2005.

[29] L. C. Mâsse, B. F. Fuemmeler, C. B. Anderson et al., "Accelerometer data reduction: a comparison of four reduction algorithms on select outcome variables," Medicine and Science in Sports and Exercise, vol. 37, no. 11, supplement, pp. S544-S554, 2005.

[30] R. Colley, S. C. Gorber, and M. S. Tremblay, "Quality control and data reduction procedures for accelerometry-derived 
measures of physical activity," Health Reports, vol. 21, no. 1, pp. 63-69, 2010.

[31] M. R. Puyau, A. L. Adolph, F. A. Vohra, and N. F. Butte, "Validation and calibration of physical activity monitors in children," Obesity Research, vol. 10, no. 3, pp. 150-157, 2002.

[32] J. F. Sallis, K. Patrick, E. Frank, M. Pratt, H. Wechsler, and D. A. Galuska, "Interventions in health care settings to promote healthful eating and physical activity in children and adolescents," Preventive Medicine, vol. 31, supplement 2, pp. S112-S120, 2000.

[33] I.-M. Lee, K. M. Rexrode, N. R. Cook, J. E. Manson, and J. E. Buring, "Physical activity and coronary heart disease in women: is "No Pain, No Gain" passé?" Journal of the American Medical Association, vol. 285, no. 11, pp. 1447-1454, 2001.

[34] C. Y. Jeon, R. P. Lokken, F. B. Hu, and R. M. Van Dam, "Physical activity of moderate intensity and risk of type 2 diabetes: a systematic review," Diabetes Care, vol. 30, no. 3, pp. 744-752, 2007.

[35] M. Tanasescu, M. F. Leitzmann, E. B. Rimm, W. C. Willett, M. J. Stampfer, and F. B. Hu, "Exercise type and intensity in relation to coronary heart disease in men," Journal of the American Medical Association, vol. 288, no. 16, pp. 1994-2000, 2002.

[36] H. D. Sesso, R. S. Paffenbarger Jr., and I.-M. Lee, "Physical activity and coronary heart disease in men: the Harvard Alumni Health Study," Circulation, vol. 102, no. 9, pp. 975980, 2000.

[37] F. B. Hu, R. J. Sigal, J. W. Rich-Edwards et al., "Walking compared with vigorous physical activity and risk of type 2 diabetes in women: a prospective study," Journal of the American Medical Association, vol. 282, no. 15, pp. 1433-1439, 1999.

[38] O. T. Raitakari, S. Taimela, K. V. K. Porkka et al., "Associations between physical activity and risk factors for coronary heart disease: the cardiovascular risk in young finns study," Medicine and Science in Sports and Exercise, vol. 29, no. 8, pp. 10551061, 1997.

[39] O. T. Raitakari, K. V. K. Porkka, L. Räsänen, and J. S. A. Viikari, "Relations of life-style with lipids, blood pressure and insulin in adolescents and young adults. The cardiovascular risk in young finns study," Atherosclerosis, vol. 111, no. 2, pp. 237246, 1994.

[40] A. Sääkslahti, P. Numminen, V. Varstala et al., "Physical activity as a preventive measure for coronary heart disease risk factors in early childhood," Scandinavian Journal of Medicine and Science in Sports, vol. 14, no. 3, pp. 143-149, 2004.

[41] O. T. Raitakari, S. Taimela, K. V. K. Porkka, and J. S. A. Viikari, "Effect of leisure-time physical activity change on high-density lipoprotein cholesterol in adolescents and young adults," Annals of Medicine, vol. 28, no. 3, pp. 259-263, 1996.

[42] K. Patrick, J. F. Sallis, J. J. Prochaska et al., "A multicomponent program for nutrition and physical activity change in primary care: PACE+ for adolescents," Archives of Pediatrics and Adolescent Medicine, vol. 155, no. 8, pp. 940-946, 2001.

[43] M. T. Story, D. R. Neumark-Stzainer, N. E. Sherwood et al., "Management of child and adolescent obesity: attitudes, barriers, skills, and training needs among health care professionals," Pediatrics, vol. 110, no. 1, pp. 210-214, 2002.

[44] R. F. Kushner, "Barriers to providing nutrition counseling by physicians: a survey of primary care practitioners," Preventive Medicine, vol. 24, no. 6, pp. 546-552, 1995.

[45] M. Sharma, "School-based interventions for childhood and adolescent obesity," Obesity Reviews, vol. 7, no. 3, pp. 261-269, 2006.
[46] M. C. Nelson, D. Neumark-Stzainer, P. J. Hannan, J. R. Sirard, and M. Story, "Longitudinal and secular trends in physical activity and sedentary behavior during adolescence," Pediatrics, vol. 118, no. 6, pp. e1627-e1634, 2006.

[47] D. Neumark-Sztainer, M. Story, P. J. Hannan, and J. Rex, "New Moves: a school-based obesity prevention program for adolescent girls," Preventive Medicine, vol. 37, no. 1, pp. 41-51, 2003.

[48] M. J. Müller, I. Asbeckl, M. Mast, K. Langnäse, and A. Grund, "Prevention of obesity-more than an intention. Concept and first results of the kiel obesity prevention study (KOPS)," International Journal of Obesity, vol. 25, supplement 1, pp. S66-S74, 2001.

[49] L. H. Epstein, R. A. Paluch, C. C. Gordy, and J. Dorn, "Decreasing sedentary behaviors in treating pediatric obesity," Archives of Pediatrics and Adolescent Medicine, vol. 154, no. 3, pp. 220-226, 2000. 